\title{
Re-Interpretation of Mathematical Literacy Based on the Teacher's Perspective
}

\section{Uba Umbara}

School of Postgraduate Studies, Universitas Pendidikan Indonesia, \& Department of Mathematics Education, STKIP Muhammadiyah Kuningan, Indonesia, uba.bara@upi.edu

\section{Didi Suryadi}

Prof., Department of Mathematics Education, Universitas Pendidikan Indonesia, Indonesia, ddsuryadil @gmail.com

This study was aimed to explore the teacher's knowledge and understanding of mathematical literacy, especially on the PISA survey, understanding of mathematical literacy, aspects, and processes of evaluating mathematical literacy in the PISA survey and the learning process carried out by respondents in their respective classes. The research used a qualitative method with case study design. The respondents involved in this study were 20 teachers. Questionnaires and interview guidelines were adopted as the instruments. The collected data were then analyzed descriptively. The results of this study shown that: (1) there were 60\% respondents have lack of knowledge on mathematical literacy, (2) around 3.63\% respondents shown the highest percentage of aspects of the learning process carried out in the class, while (3) aspects of mathematical literacy assessment process in the PISA survey the lowest percentage (2.47\%). The data indicated that respondents understand the learning process better than the process of assessing mathematical literacy in the PISA survey. Campaigning for mathematical literacy in various teacher communities can be carried out simultaneously, structured, and systematically through a special program and continues to look at its development, with the intention of the teacher has a target in developing students' mathematical literacy and to improve Indonesia's ranking in the PISA survey.

Keywords: mathematical literacy, teacher's perspective, quantitative literacy, numeracy literacy, PISA survey, learning process, assessment process

\section{INTRODUCTION}

Someone is often trapped in a formal process and rigid rules in learning math. The vague structures on it must be studied in a manner structurally. Brownell (in Reys et al., 1998) stated that mathematics can be viewed as a system that consists of ideas,

Citation: Umbara, U., \& Suryadi, D. (2019). Re-Interpretation of Mathematical Literacy Based on the Teacher's Perspective. International Journal of Instruction, 12(4), 789-806. https://doi.org/10.29333/iji.2019.12450a 
principles and processes thus the linkage among these aspects must be built and emphasis on the aspects of reasoning or intelligence not on children memorization. However, the transformation of thinking skills from concrete to abstract or vice versa has become a unique thing in learning math. Three main phases that can be seen in the performance of mathematical tasks: translating from verbal problem statements to mathematical expressions, performing operations on expressions, and validating solutions (Gagne, 1983). Here, strengthening the role of mathematics is needed in everyday life.

Learning mathematics depends on a person's cognitive structure so that one's mathematical knowledge will be different from one another. Mathematical knowledge that belongs to a person is its tendency to respond to perceived mathematical problem situations by reflecting on their problems and solutions in social contexts and by developing mathematical actions, processes, and objects (Dubinsky, 2001). In this case, the role of mathematics can be a solution to solve everyday life problem contextually. Mathematics is a tool for fulfilling individual needs. So, if mathematics has such social and cultural interests, we need to consider what mathematics should be learned in school and how to teach it (Noyes, 2017). The implications of these principles are significant for learning mathematics in schools, especially regarding the usefulness of mathematics. It shows that mathematics education is one of the important foundations of science in education (Umay, 2003).

The contribution of mathematics education at least can be viewed from three things, i.e. from the needs of child development, society and the real world of work (Suryadi, 2012). The real contribution of mathematics education sometimes cannot be optimized due to the burden of learning mathematics in school especially in Indonesia which is felt too heavy. It is important to notice that mathematics that we learn and the mathematics that we need to know are two different things. Its differences situated in the fact that not all mathematical content that we know as students can be applied in our daily lives (Ojose, 2011). On the other hand, Suharta \& Suarjana (2018) stated that, mathematical literacy must be the most important part of mathematics learning. For this reason, Indonesia needs to start a campaign on mathematical literacy systematically and structured through a government policy package program since Indonesia's participation in PISA is still unsatisfied yet.

The author believes that the essential main study which requires to be done before looking at the mathematical content taught at school is to look at the mathematics teacher first. The author assumes that mathematics teachers in Indonesia may not have received enough information about the PISA survey. Gallimore and Tharp (in Draper, 2002) stated that schools must teach students to be literate in the most general sense of being able to read, write, speak, count, reason, and manipulate symbols and verbal and visual concepts. We need to understand how important the contribution of mathematical literacy is to the students and the perceptions of mathematics teachers especially in daily life (Venkat, 2010).

Indeed, in this article, the researcher tried to explain whether the respondents were aware of the PISA survey? Did the respondent know the aspects and process of 
assessing mathematical literacy in the PISA survey? What is the learning process carried out by respondents in each class?

These questions seem simple but fundamental. They need to be answered in order to determine the policy package on mathematical literacy, which in the future can improve the results of the PISA survey, especially on improving Indonesia's ranking. This study was conducted as feedback for teachers in enhancing the learning process in which expedient to help teachers improve the learning process, they need in concepts that need improvement (Al-Hattami, 2019).

This research became the starting point for respondents about what and how mathematics was taught in their class related to mathematical literacy specifically and the role of mathematics in general. We try to provide an understanding of the importance of campaigning mathematical literacy as a structured and systematic movement, especially in the Indonesian Ministry of Education and Culture. This movement is important to do, considering that literacy plays an important role in the lives of students in the future. Re-interpretation of mathematical literacy for teachers is intended so that teachers understand the true meaning of mathematical literacy, both its meaning and implementation, which depends on aspects, contexts, and processes. Reinterpretation of mathematical literacy is important so that the understanding of teacher mathematical literacy is the same as literary sources that match PISA interpretations and experts. So, in this article, we provide a perspective on mathematical literacy based on history, definitions, types, components and basic competencies in mathematical literacy.

\section{LITERATURE REVIEW}

\section{The History of Mathematical Literacy}

Mathematical literacy has been a trending issue lately which was known as quantitative literacy. Steen (2001) shown that there are small but important differences in some definitions that exist and, although he does not suggest phrases as definitions, refers to quantitative literacy as the capacity to effectively deal with quantitative aspects of life. Steen himself adopted the concept of Cremin. Literacy is clearly a complex problem rather than a simple one. Most existing Steen definitions mention, giving explicit attention to numbers, arithmetic, and quantitative situations (de Lange, 2003). The absence of actual meaning about literacy does not mean that literacy does not have a clear reference.

In this case, let's take a look at the quantitative literacy definition of NCES (1993) that the knowledge and skills needed to implement arithmetic operations, either alone or sequentially, use numbers embedded in printed material (for example, balancing checkbooks, completing order forms). The meaning of literacy seems to be limited to numerical skills which are limited to mathematical operations. It does not mean, the need for limited mathematics is a major factor in defining such literacy. In its development, NCES (2005) defined two phrases, namely literacy, and enumeration. Literacy was defined as the knowledge and skills needed to understand and use information from the text and other written formats, while enumeration applies to the knowledge and skills needed to manage the mathematical demands of various situations. In fact, Cremin (Jablonka, 2003) stated that numerical interpretation is not only about 
critical ideas in evaluating the use and misuse of data and numbers, but a capability that must be seen as the most important way of liberating the illiterate of an individual.

In particular mathematical literacy was interpreted as an individual's ability to solve a situation related to mathematics. The National Council on Education and the Disciplines (Steen, 2001) prefered to talk about 'quantitative literacy' in emphasizing the importance of investigating the meaning of numeracy in the society which continues to increase the use of numbers and quantitative information. So he underlined the role played by numbers and data in contemporary society is almost endless. The temporary conclusion that we get from the two terms used, both as quantitative literacy and numeracy literacy lies in the arithmetic aspects used by individuals which are still seen as simple definitions of mathematics related to daily activities.

This corresponds to the term that appears first in Webster's Collegiate Dictionary, that enumeration is characterized by the capacity to think and express quantitative (Jablonka, 2003). However, limited mathematics is still identical with quantitative problems, numbers, and data. Thus, literacy is focused on both of these things, not in a more general form. In other words, quantitative literacy and numeracy literacy are only part of mathematical literacy. It needs to be realized that a lot of mathematical literacy, numeracy, and quantitative literacy are interpreted into various languages so that the meaning must be paraphrased (Jablonka, 2003).

As an elaboration of several studies, many use these both terms, especially to describe the content and role of mathematics. Based on this, we are faced with the need to define literacy in a broad sense. Describing mathematical literacy requires not only a broader definition but also attention to changes in other school disciplines (de Lange, 2003). The existence of mathematical interconnection with other disciplines must also be a focus in understanding mathematical literacy.

By looking at this issue, comprehensively it would be more ideal if we looked at the development of mathematical literacy issues discussed by the OECD considering that in the following period, the OECD was always a reference to the notion of mathematical literacy. OECD's particular understanding of mathematical literacy has undergone three changes including assessment frameworks 1999 \& 2003, assessment framework 2006 \& 2009 , and assessment framework $2013 \& 2107$. To facilitate writing, we mention it with the first, second and third periods. There is no significant change from the definitions listed in the first and second periods. The difference is only in using and utilizing mathematics by someone to follow through their daily needs as citizens. Where we know that the notion of literacy in the first OECD period only uses terminology to use (see OECD, 1999, 2003, 2006 \& 2009).

The radical differences we call it to occur in the notion of mathematical literacy in the third period (see OECD, 2013 \& 2017). This third definition, it seems very prominent in the function of mathematical literacy for someone. In the initial editorial, it is very clearly stated the individual's ability to formulate, employ, and interpret mathematics in various contexts. This strengthens the position of literacy, with not only the role of recognizing the ability of individuals to recognize and understand the role of mathematics but also more about how an individual can interpret and articulate 
mathematics in more complex contexts. In addition, in this third period, the demands of literacy in terms of competencies shifted from providing judgments and considerations in utilizing mathematics to predict the phenomenon of situations faced by an individual. In our opinion, this is important considering the role of mathematics can not only be used in observing the occurrence situation.

In the development of the OECD definition remains a general consensus about the definition of the concept of mathematical literacy in research on mathematics teaching and learning, but there is uncertainty about the highlighted in the interpretation of the OECD definition and the application to the development of student mathematical literacy (Haara et al., 2017). Thus, up to this moment, there have been no clear recommendations regarding alternative ways that mathematical educators can take in developing mathematical literacy. The positive side that can be taken situated in the flexibility of ways that can be taken to develop literacy itself based on the basic concepts of mathematical literacy itself.

The general view of mathematics as a tool describes the importance of having mathematical knowledge. Dubinsky (2001) stated that mathematical knowledge is an individual tendency to describe various contexts of mathematical problems faced by developing analysis and synthesis actions on problems involving mathematical processes and objects to find solutions to the mathematical problems they cope with. The process of obtaining mathematical knowledge can be understood as a structured sequence starting from interpreting mathematical problems through the process of internalization to the process of externalizing the concepts needed to solve the problem.

The mathematical process seems to be the OECD's main reference for interpreting literacy models in mathematical practice. Mathematical literacy as the main issue places the importance of mathematics as a basis for individuals in society. Noyes (2017) stated that mathematical thoughts and considerations needed by citizens in the future that are developing and changing must be supported by the curriculum and how to teach mathematics itself. This is then the function played by mathematical literacy, which becomes important in mathematical activities. Literacy and literacy learning are important parts of mathematics learning (Draper, 2002).

The importance of mathematical literacy as the main tool for someone to live their daily lives is a top priority to continue to develop. It is indisputable that in today's society the ability to deal with numbers and interpret quantitative information becomes an important component of literacy in addition to speaking, writing and reading (Jablonka, 2003). As explained in the previous section, before the 21 st-century quantitative literacy was known to contribute to the quantitative aspects of life. Its role might be to limit the usefulness of mathematics as a problem-solving tool in everyday life. The National Adult Literacy Survey (NCES, 1993) stated the knowledge and skills needed to implement arithmetic operations, either alone or sequentially, using numbers (de Lange, 2003).

However, it indicated that mathematical literacy is not limited to the ability to apply quantitative aspects of mathematics but involves mathematical knowledge in a broad and comprehensive sense from several parts, namely spatial literacy, numeracy, and 
quantitative literacy. De Lange (2003) then described the correlation between mathematical literacy and other parts of literacy based on the phenomenological category, which clearly shows the differences from the terms described previously, mathematical literacy consists of spatial literacy (space and shape), numeracy (quantity), and quantitative (quantity, change and relationships, and uncertainty).

Mathematical literacy encompasses meaning not only synonymous with applying arithmetic operations but also takes a role in solving problems of daily life in combination with mathematical conceptuality which consists of the types of mathematical literacy above. On the other hand, Ojose (2011) stated that mathematical literacy does not imply detailed knowledge in the fields of calculus, differential equations, topology, analysis, linear algebra, abstract algebra, and other complex mathematical concepts, but what mathematics can achieve through understanding and a broad appreciation of mathematics itself. Meanwhile, Jablonka (2003) took a broader term by stating that the main purpose of literacy is to see the world through mathematics so that literacy places mathematics as a high-level thinking activity encompassing problem-solving abilities.

The focus in mathematical literacy situated in the way one uses conceptual mathematical knowledge and abilities in a variety of social contexts. Gilbert (in Mkhwanazi \& Bansilal, 2014) stated that context expresses about coherence, connections and/or relationships which function to describe and give meaning to words, phrases, and sentences. Duranti \& Goodwin (1992), identified four contexts namely contextual settings, environmental behaviour, language usage, and extra situational knowledge background. Contextual settings refer to the interaction of social and spatial settings, environmental behaviour refers to the ability to predict what will happen by setting preconditions for coordinated social action, the use of language refers on how to communicate, while background situational knowledge refers to other knowledge outside the context that supports another context.

This implies that mathematical literacy implies a person's ability to analyze and communicate mathematical ideas. In other words, an important element in the process of mathematical literacy is the internalization of mathematical concepts and externalization of contexts which play a role in solving mathematical problems. Mathematical literacy is defined more broadly by OECD (2013 \& 2017) as the ability of individuals to form, employ and interpret various mathematical contexts to interpret phenomena in social life so as to help individuals recognize the role that mathematics plays in their lives as constructive citizens, involved and reflective.

Literacy which is operationally defined by OECD does not only describe mathematical literacy which plays a role in the concepts and structure of mathematics but also further describes mathematics as a procedure and tool in applying mathematics. Mathematical literacy is a subject that is specifically driven by the application of mathematics in life (Julie, 2006). From this side, someone who studies mathematics is possible to possess and develop the ability to think critically and systematically in interpreting a situation through mathematical and mathematical modelling. This is the historical basis of the PISA framework which includes mathematical concepts, procedures, facts and tools 
actively to formulate, employ and interpret mathematics in various contexts (OECD, 2017). However, Rico (in Sáenz, 2009) explained that PISA is not related to the mathematics curriculum taught, but revolves around the right and coherent part of the functional model of mathematics learning which consists of contextual instruction by creating problem situations, conceptual procedures that are mathematical content needed to solve problems, cognitive subjects that activate competencies to connect the real world where problems are generated by the mathematics needed to solve them.

This concept is then seen as intersecting with realistic mathematical concepts that are the source and domain of the application of mathematics (Gravemeijer, 1994). This is similar to De Lange (in Stacey, 2011) who stated that the concept of mathematical literacy is closely related to several other concepts discussed in mathematics education, especially regarding the processes and components in mathematical and mathematical modelling. The mathematical process can be interpreted as a cycle that simultaneously explores the role of one's mathematical knowledge in integrating between the real world and the world of mathematics which plays a role in solving problems. The real-world context in this conception certainly intersects with the cultural context that grows and develops in various countries.

Solomon (2009) interpreted theories and practices that recognize the role of mathematics as a social and cultural activity involving the role of language, which focuses on mathematical language, understanding basic rules, and sociological cultural contexts as tools in mathematics learning. The capacity of mathematical literacy as a tool that is in a social context places the function of mathematical literacy that specifically applies to certain fields according to the individual's own needs. Every effort to define mathematical literacy will be faced with problems that cannot be conceptualized as mathematical knowledge specifically in terms of individual capacity to use and apply it, thus functionally mathematical literacy only applies to situations where this knowledge will be used by the individual (Jablonka, 2003).

Thus, a person's mathematical literacy abilities differ from one another in certain contexts, as the mathematical literacy possessed through the competencies and certain mathematical skills that they learn. Earned (in Ic \& Tutak, 2018) mentioned some of the metamorphic literacy skills and competencies such as mathematical operations, conceptualization in mathematical thinking and knowledge practiced in various mathematical content. The complexity of mathematical content, in the form of new knowledge and old knowledge possessed by students in the cognitive structure of students, has a central role in the development of students' own mathematical literacy. Mathematical literacy plays a role in gaining new knowledge obtained by students by using real-life situations that are done through information processing in a concrete way (Spangenberg, 2012). Kramarski \& Mizrachi (2006) described it as a combination of all types of mental representations that play a role in supporting the development of mathematical literacy.

The fundamental difference between mathematics and mathematical literacy positioned in the content and context studied. Mathematics works with content that includes concepts and procedures that tend to be very technical, while mathematical literacy 
works based on contexts that tend to lead to application and practicality. In particular, Sfard (Venkat et. al., 2009) noted that mathematical literacy placed between everyday life and mathematics that promotes thinking as a form of communication that consists of asking questions, hypothesizing, finding arguments and drawing conclusions in a situation. The practical and application context, is a priority in mathematical literacy, although it may tend to limit the space contained in mathematics itself. Like Dowling, which mentioned the exclusive or special participation of contextual mathematical activities inhibits mathematical understanding and partially only provides a level of preparation for life which is limited to certain contexts (North \& Christiansen, 2015).

The consequences of mathematics taught contextually according to Dowling (in North \& Christiansen, 2015) focusing solely on general relevance are considered to only be learned by students who have lower mathematical abilities while abstract mathematics can be learned by students with higher abilities. This opinion contradicts with Draper (2002) which stated that the final benefit of this adaptation is active and pleasant mathematics class based on a constructivist understanding by accommodating students' needs and involving communication between students and teachers. With the diversity of mathematical literacy definitions, we must finally realize that there is no consistent definition of mathematical literacy. So, the opinion of Steen (2001) who declared that literacy cannot have a permanent and constant meaning throughout time and place, can be a benchmark in respecting any differences that arise in describing literacy.

\section{Basic Competency for Mathematical Literacy}

The need for mathematical literacy will differ between individuals and groups, according to their individual needs, both in terms of content and the mathematical context itself. Perspectives on different mathematical literacy procreate a conception of the individual's perspective on mathematical literacy itself, mainly relying on the usefulness of mathematical literacy in practical and applicable ways. As with Niss, Blum \& Galbraith (in Mumcu, 2016), the intended application is used to show various situations in merging mathematics and real life. Using mathematical literacy for certain needs requires understanding basic mathematical concepts that relate to each other even in different mathematical situations.

The literacy of mathematics plays a role more than just understanding mathematical ideas; this involves basic literacy and an independent ability to use mathematical thinking, build understanding, and solve problems (Yore, 2007). This description of literacy tends to reveal an orientation of life preparation that depends on the contextualization of everyday life situations (Venkat, 2010). This is a general content in developing mathematical literacy. Thus, designed for this purpose, describing the basic competencies of mathematical literacy is a must.

Bowie and Frith (in Bansilal \& Debba, 2012) shown that from several definitions of mathematical literacy, he set out four elements of mathematical literacy which consist of content, context, abilities, and behaviours that will be used in using mathematical literacy as an ability. Meanwhile, Graven \& Venkat (2007) identified the main factors in mathematical literacy is the nature and focus of the relationship between content and context. Prominence on the importance of determining the basic competency 
components of mathematical literacy was de Lange (2003), to have mathematical literacy, individuals need all mathematical competencies in various levels and trust in their own ability to use mathematics and explore quantitative ideas from a historical, philosophical point of view, and social.

Descriptions of basic competencies in developing mathematical literacy are needed to provide a reference to the teacher in teaching mathematics in the classroom. Kilpatrick (2001) called it the five elements of mathematical abilities, including (a) conceptual understanding; (b) procedural fluency; (c) strategic competencies; (d) adaptive reasoning; and (e) productive dispositions. Kilpatrick took the term mathematical ability, as, from the terms of mathematical literacy, numeracy, mastery of mathematics, and mathematical competencies discussed were not suitable to interpret the success of learning mathematics. The five elements of mathematical ability are a unit that emphasizes the importance of conceptuality, procedural, competency in mathematical activities, thinking capacity and individual perspective on the usefulness of mathematics.

On the other hand, Carpenter and Lehrer (in Draper, 2002) classified it through mental activity in understanding mathematics which contains five aspects, i.e.: (a) building relationships, (b) expanding and applying mathematical knowledge, (c) reflecting experience, (d) articulate what is known, and (e) make their own mathematical knowledge. Through understanding the mathematics that arises from the five forms of mental activity, it provides an understanding that literacy learning is integrated with mathematics learning. Meanwhile, PISA (OECD, 2006 \& 2009) uses eight competencies in mathematical literacy compiled based on the formulation of Niss (1999) and Neubrand et al, (2001), namely:

a. Thinking and reasoning. Emphasizing the ability to distinguish definitions, theorems, guesses, hypotheses, examples, conditioned statements and understand and deal with the extent and limits of the mathematical concepts given.

b. Argumentation. Emphasizing the ability to involves several types of mathematical reasoning; heuristic mathematical argumentation, creating and expressing mathematical arguments.

c. Communication. Emphasizing the ability to express themselves in communicating mathematical concepts and content in a certain way.

d. Modeling. Emphasizing the ability to compile, interpret, validate, reflect, analyze and control the mathematical modeling of contextual problems into the structure of mathematics.

e. Problem posing and solving. Emphasizing the ability to formulate and solve various types of mathematical problems in numerous ways.

f. Representation. Emphasizing the ability to interpret various information in the form of representations of objects and mathematical situations so that interactions occur between various representations based on specific situations and objectives.

g. Using symbolic, formal and technical language and operations. Emphasizing the ability to interpret, decode, operate and understand the relationships between various symbolic and formal languages in solving equations and doing calculations. 
h. Use of aids and tools. Emphasizing the ability to use knowledge in utilizing the functions of various tools and the limitations of these tools in mathematical activities.

The OECD then made partial changes in the definitions and basic competencies of mathematical literacy in the future (OECD, 2013 \& 2017) describing seven basic mathematical abilities used in namely:

a. Communication. Emphasizing the ability of individuals to present problems and solutions for others.

b. Mathematizing. Emphasizing the ability to transform problems in the real world into mathematical form through mathematical modeling.

c. Representation. Emphasizing the ability to interpret various mathematical representations, both objects, and mathematical situations.

d. Reasoning and argument. Emphasizing the ability to think logically in exploring and connecting the elements of problems and their solutions.

e. Devising strategies for solving problems. Emphasizing the ability to choose and use various strategies in solving problems mathematically.

f. Using symbolic, formal and technical language and operations. Emphasizing mathematical literacy skills in understanding, interpreting, manipulating, and utilizing symbolic expressions in various mathematical contexts in solving mathematical problems.

g. Using mathematical tools. Emphasizing the ability to use mathematical tools to help with mathematical activities.

This partial change regarding the basic competencies of mathematical literacy provides an understanding that mathematical literacy cannot have a fixed component because of the mathematical needs that develop over time. As we know that social and environmental situations change, making mathematical literacy varies at least in the historical period and background of a culture (Steen, 2001). This change in the formulation of the OECD mathematics literacy competency is based on the different level of proficiency of the previous survey.

\section{METHOD}

\section{Research Design}

This research basically aims to provide an overview of the knowledge of junior high school mathematics teachers about mathematical literacy, especially regarding surveys conducted by PISA, including analyzing their knowledge of the mathematics domain which consists of mathematical processes, knowledge of mathematical content and context use. Therefore, the research design used was qualitative with a descriptive study. The sampling technique was purposive sampling, on the basis of our judgment of their typicality or possession of the particular characteristics being sought to satisfactory to our specific needs. The research respondents consisted of 20 math teachers comprising 8 men and 12 women. They are teachers of the Mathematics Subject Teachers' Community members in Kuningan District. 


\section{Instruments and Data Collection Techniques}

The instruments used to obtain research data were questionnaires and interview guidelines. The questionnaire used a closed questionnaire, while the in-depth interview conducted to verify the respondent's answer from the questionnaire has been filled. The procedures carried out in this study were as follow:

a. This research was conducted on November 24, 2018, during a routine meeting they conducted with their group.

b. Respondents were given 30 minutes to answer 20 questions.

c. After the respondent finished filling out the questionnaire, a structured and in-depth interview was carried out in a closed room.

Scoring rubric was done based on rubric holistic scoring, i.e. scoring was carried out on the whole process without assessing component parts separately. While the indicators of the questions posed in the questionnaire and interviews were divided into 2 parts, namely: knowledge of the PISA survey and knowledge of PISA assessment both in terms of understanding the PISA survey, aspects and processes of mathematical literacy assessment in the PISA survey and the learning process carried out by respondents in each class. The assessment guidelines were carried out based on the following holistic assessment rubric.

Table 1

Template for Holistic Rubrics

\begin{tabular}{cl}
\hline Score & Notes \\
\hline 5 & Showing a complete understanding of the problem. \\
4 & Showing enough understanding of the problem. \\
3 & Showing only a partial understanding of the problem. \\
2 & Showing a little understanding of the problem. \\
1 & Showing no understanding of the problem \\
0 & There is no answer / No effort \\
\hline
\end{tabular}

\section{Data Analysis Technique}

Data analysis techniques were carried out descriptively. The classification conversion guidelines were made using the modified benchmark norms from the Ganesha University of Education Handbook (Suharta \& Suarjana, 2018) as follows:
a. If average score $>80 \%$ then it is classified high
b. If $65 \%<$ the average score of $\geq 80 \%$ then it is classified medium
c. If the average score $\leq 65 \%$ then it is classified low.

\section{FINDINGS}

The number of question items regarding the PISA survey and mathematical literacy amounts to 20 . The scores of each item are 5, so the maximum score is 100 . The results of the questionnaire and interview are presented in the following table 2. 
Table 2

Holistic Scoring Rubric

\begin{tabular}{llllll}
\hline Descriptive Statistics & Gender & \multicolumn{3}{l}{ Knowledge } \\
\cline { 2 - 6 } & Male & Female & High & Medium & Low \\
\hline Maximum Score & 77 & 80 & 80 & 63 & 55 \\
Minimum Score & 41 & 36 & 77 & 66 & 36 \\
Mean & 53,75 & 52,50 & 78,33 & 59,40 & 44 \\
Deviation Standard & 11,93 & 15,38 & 1,53 & 2,61 & 6,55 \\
$\mathrm{~N}$ & 8 & 12 & 3 & 5 & 12 \\
Percent & 40 & 60 & 15 & 25 & 60 \\
Classification & Low & Low & Medium & Low & Low
\end{tabular}

In accordance with Table 2 above, male respondents had a higher average score of 53.75 than female respondents at 52.50 , but based on the highest score female respondents were higher than male respondents, 80 to 77 . This shows that male respondents gave a higher response to some statements given by showing a fairly complete understanding of the problems raised in the questionnaire. Meanwhile, based on knowledge scores, it can be concluded that the majority of respondents have low knowledge of mathematical literacy, which is $60 \%$. By gender, the classification of mathematical literacy is at a low level. However, there is one medium level based on mathematical literacy knowledge in which reached the high classification.

To show a comprehensive analysis on how respondents' comprehension of mathematical literacy in terms of understanding of the PISA survey, aspects and processes of evaluating mathematical literacy in the PISA survey and the learning processes carried out by respondents in the class are presented in table 3 below.

Table 3

Score based Indicator

\begin{tabular}{|c|c|c|c|}
\hline \multirow{4}{*}{$\begin{array}{l}\text { Descriptive } \\
\text { Statistics }\end{array}$} & \multicolumn{3}{|l|}{ Indicator } \\
\hline & Understandin & Aspects and Processes of & The Learning Process \\
\hline & $\mathrm{g}$ of The PISA & Mathematical Literacy & Carried Out By Teachers \\
\hline & Survey & Assessment Carried Out by PISA & In Each Class \\
\hline Maximum Score & 5 & 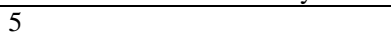 & 5 \\
\hline Minimum Score & 1 & 1 & 1 \\
\hline Mean & 2,49 & 2,47 & 3,63 \\
\hline Deviation Standard & 1,22 & 0,78 & 0,78 \\
\hline
\end{tabular}

In accordance with table 3 above, the aspects of the learning process carried out by respondents in the class had the highest percentage of 3.63 while the aspects of the process of assessing mathematical literacy in the PISA survey had the lowest percentage of 2.47. The results of interviews on this indicator item showed that most respondents did not understand the PISA survey even some respondents did not know the survey at all. Furthermore, indicators on aspects of understanding of the PISA survey and aspects and processes of mathematical literacy assessment address respondents' insufficient knowledge about the PISA survey and the model on the practice of PISA assessment which consists of mathematical content categories, real-world context categories, mathematical thinking and actions, basic mathematical competencies and process. 
This shows that respondents understand the learning process better than the process of assessing mathematical literacy in the PISA survey. Respondents received less information about the PISA survey. Based on the results of the interview, we identified two factors. First, respondents did not get information about the aspects and process of literacy assessment conducted in the PISA survey. Most respondents said that they only got information about the results of the PISA survey which placed Indonesia in a less satisfactory ranking. Secondly, respondents never tried to find information about the PISA survey. In this case, the habits and perspectives of the teacher must be changed so that they have the desire to find information about the results of research on mathematics learning and teaching so that they can implement the results of research to improve the learning that has been done so far.

For the time being, the indicators of the learning process carried out by respondents in their respective classes, we asked about whether the learning process carried out connected the context of the problem, used contexts that were close to students and conducted process assessments to see students' ability to solve problems, especially contextual problems. The results of interviews on this indicator item shown that most respondents carried out the learning and assessment process by connecting the context of the problem which is close to the students both in part and in all the concepts of the mathematics material being taught. However, it was found several respondents who still had difficulties in developing contextual-based teaching materials. Some of the factors identified include their understanding of the usefulness of mathematics in the limited context of day-to-day life, creative ideas in developing teaching materials are still unexplored, and limited learning resources.

This is alleged as respondents are accustomed to implement learning by providing the mechanical understanding and inductive understanding to students in learning. Where the learning process is only directed at the activities of remembering, calculating, and applying a concept or formula simply in some similar cases. Giving a stimulus to prove, estimate the truth, analyze analytically in mathematical concepts is still not optimal. Another fact, we find that the interpretation of mathematical literacy is still simple and limited to the individual's ability to read, write, count, how to learn mathematics, and apply concepts learned for practical use. So, it is necessary to re-interpretation mathematics literacy.

\section{DISCUSSION}

The low level of knowledge and understanding of respondents about the PISA survey and the aspects as well as processes of mathematical literacy assessment indicate a lack of information received or accessed by respondents. By paying attention to the data support for the average value of male respondents that is greater than the female respondents, we believe that male respondents tend to better understand the problems raised in the questionnaire. We suspect that male respondents have a higher interest in accessing information about developments in mathematics learning and literacy. General evidence shows that women do have a somewhat more negative attitude towards mathematics than men (Benbow, 1988). Overall, we identified based on interviews conducted, the low level of knowledge about mathematical literacy was due to the lack 
of socialization from policymakers about mathematical literacy. In addition, respondents also rarely accessed information about the PISA program so that knowledge of the model in PISA assessment practices consists of categories of mathematical content, realworld context categories, mathematical thinking and actions, basic mathematical competencies and processes.

However, this study implies that teachers are accustomed to implement learning based on real-world contexts even though the learning that has been carried out has not been maximized. Especially related to the categories of mathematical content, basic mathematical competencies, and mathematical processes. Most of the respondents had a lack of understanding regarding this matter, implying that the learning done was not specifically carried out to develop mathematical literacy. In most countries, the gap between mathematics and mathematical literacy and an understanding of what constitutes mathematical literacy is hardly recognized (de Lange, 2003).

Some respondents admit that sometimes when they are faced with the problem of the amount of material that has not been taught, they tend to carry out learning with traditional approaches. Overall, they have not realized the importance of mathematical literacy so that the learning done has not been carried out based on important aspects of mathematical literacy. In fact, it cannot be denied that in today's society the ability to use numeracy and interpret quantitative information is an important component of literacy (Jablonka, 2003). Three important aspects presented in PISA (content, process, and context) need to be considered in developing curriculum and implementing mathematics learning in class in an essential manner by maximizing the reasoning and communicating process especially in solving problems related to life. Literacy is at the centre of many educational reforms and policy initiatives so that it becomes an important element of teacher preparation in carrying out learning in the classroom (Draper et al., 2012).

Looking at the results of the research described earlier, it is important for education policymaker from the centre to the regions to provide comprehensive information on mathematical literacy. So that the teacher has knowledge and understanding of mathematical literacy. Clear information about the essence of mathematical literacy, especially regarding mathematical literacy content can reduce resistance to teaching theory and practice in the implementation of learning. The demands made to develop mathematical literacy can be seen as an aggressive emphasis for teachers (Draper et al., 2012). Meanwhile, the findings in Nel (2012) focused on promoting mathematical literacy, especially on mathematical literacy content and mathematics teachers can help to better develop teachers.

The general message of the PISA study for Indonesia indicated that students are not accustomed to solve non-routine problems, weak in modelling real situations into mathematical problems and interpreting mathematical solutions to real-world situations and reasoning, interpreting and deficient in analyzing. By looking at the results of this study, it revealed that respondents have a lack of understanding on the aspects and processes of evaluating mathematical literacy. It shows the importance of providing an understanding of the fundamental differences regarding mathematical and pure 
mathematical literacy. De Lange (in Gatabi et al., 2012) exposed that even though pure mathematics is very important for doing mathematical activities, it is not enough to carry out these activities in the real world.

The campaigning for mathematical literacy as an important competency was considered to be very important for respondents to have a foothold in carrying out the mathematics learning process, in order to maximize the potential of students in developing mathematics literacy. This is in accordance with Colwell \& Enderson (2016) who stated that to prepare respondents to enter the 21 st-century class, we must explain practices that include strategies and reinforcement of mathematical literacy to assist in exploring, resolving and reflecting on real problems in mathematics. Possible efforts can be made is to develop a math curriculum which accommodates mathematical literacy. In fulfilling future demands and preparing students, the mathematics literacy curriculum is important to be developed as a bridge between school and society, the focus of developing mathematical literacy that must be developed is an authentic and relevant real-life context in learning (Botha \& Van Putten, 2018).

Based on our study, mathematical literacy can be interpreted as an individual's ability to formulate, employ, interpret, decode, articulate, and predict mathematics against the phenomenon of situations encountered in various complex contexts that require highlevel thinking activities. This is different from the respondents' comprehension who only know the meaning of simpler mathematical literacy. Mathematical literacy does not imply detailed mathematical concepts in the fields of high-level mathematics such as calculus, differential equations, topology, analysis, linear algebra, abstract algebra, and other complex mathematical concepts, but what can be achieved by mathematics through understanding and appreciation broad about mathematics itself (Ojose, 2011), but needs to be given special attention to changes in other school disciplines (de Lange, 2003), with the aim that individuals have the ability to see the world through mathematics (Jablonka, 2003). High-level thinking activities involved in literacy can be trained by designing functional learning models by maximizing instruction and contextual procedures that enable competencies to connect the real world with the world of mathematics.

\section{CONCLUSION}

Based on the description of the results, it can be concluded that:

a. The respondents' truncated knowledge of the survey conducted by PISA can be the basis for the start of a campaign about programs in various teacher communities. In general, this campaign was carried out with the aim of improving Indonesia's ranking in the PISA survey, specifically to develop students' mathematical literacy in preparing for their lives and their future.

b. The respondents' comprehension of the aspects and processes of evaluating mathematical literacy is the main thing that must be developed in a structured and systematic manner. It is intended that the content, process, and context in mathematical literacy be the basis for the development of the implementation of mathematics learning. 
c. The learning process carried out by respondents in their respective classes, by connecting and using contexts that are close to students and conducting process assessments to see students' ability to solve contextual problems ought to continue to be maintained and developed.

d. The development of mathematical literacy in learning mathematics is important, especially to bridge knowledge and understanding of mathematical concepts with the usefulness of mathematics in everyday life.

In accordance with the conclusions above, it is recommended that: (1) teachers and education policymaker should continue to examine the development of mathematical literacy, especially in the fundamental aspects of basic mathematical abilities that will endure to develop in accordance with the needs and challenges of the world that prolong to grow in the future, (2) it is recommended that the Indonesian Ministry of Education and Culture be more vigorous in campaigning for mathematical literacy so that teachers can design and develop functional learning models to improve mathematics literacy, and (3) other researchers to conduct broader research with more respondents.

\section{REFERENCES}

Al-Hattami, A. A. (2019). The perception of students and faculty staff on the role of constructive feedback. Int. J. of Ins., 12(1), 885-894. doi: 10.29333/iji.2019.12157a.

Bansilal, S., \& Debba, R. (2012). Exploring the role of contextual attributes in a Mathematical Literacy assessment task. African J. of Research in Mathematics, Science and Technology Education, 16(3), 302-316. doi: 10.1080/10288457.2012.10740747.

Botha, H., \& Van Putten, S. (2018). How mathematical literacy teachers facilitate mathematization in modeling situations. African J. of Research in Mathematics, Science and Technology Education, 22(1), 93-102. doi: 10.1080/18117295.2018.1437337.

Colwell, J., \& Enderson, M. C. (2016). "When I hear literacy": Using pre-service teachers' perceptions of mathematical literacy to inform program changes in teacher education. Teaching and Teacher Education, 53, 63-74. doi: 10.1016/j.tate.2015.11.001.

De Lange, J. (2003). Mathematics for literacy. In M. Niss (Eds.), Quantitative literacy and mathematical competencies (pp. 75-89). Princeton, NJ: National Council on Education and the Disciplines.

Draper, R. J. (2002). School mathematics reform, constructivism, and literacy: A case for literacy instruction in the reform-oriented math classroom. Journal of Adolescent \& Adult Literacy, 45(6), 520-529.

Draper, R. J., Broomhead, P., Jensen, A. P., \& Nokes, J. D. (2012). (Re) imagining literacy and teacher preparation through collaboration. Reading Psychology, 33(4), 367-398. doi: 10.1080/02702711.2010.515858.

Dubinsky, E. (2001). Using a theory of learning in college mathematics courses. Coventry: University of Warwick.

Duranti, A., \& Goodwin, C. (Eds.). (1992). Rethinking context: Language as an interactive phenomenon (Vol. 11). Cambridge University Press.

Gagne, R.M. (1983). Some issue in psychology of mathematics instruction. Journal for Research in Mathematics Education. 14(1), 7-18. doi: 10.2307/748793 
Gatabi, A. R., Stacey, K., \& Gooya, Z. (2012). Investigating grade nine textbook problems for characteristics related to mathematical literacy. Mathematics Education Research Journal, 24(4), 403-421. doi: 10.1007/s13394-012-0052-5.

Gravemeijer, K. (1994). Educational development and developmental research in mathematics education. J. for Res. in Math. Edu., 25(5), 443-471. doi: 10.2307/749485.

Graven, M., \& Venkat, H. (2007). Emerging pedagogic agendas in the teaching of Mathematical Literacy. African Journal of Research in Mathematics, Science and Technology Education, 11(2), 67-84. doi: 10.1080/10288457.2007.10740622.

Haara, F. O., Bolstad, O. H., \& Jenssen, E. S. (2017). Research on mathematical literacy in schools-aim, approach, and attention. Eu. J. of Sci. and Mat. Edu., 5(3), 285-313.

Ic, U., \& Tutak, T. (2018). Correlation between computer and mathematical literacy levels of 6th grade students. European Journal of Educational Research, 7(1), 63-70. doi: 10.12973/eujer.7.1.63.

Jablonka, E. (2003). Mathematical literacy. In Second international handbook of mathematics education (pp. 75-102). Springer, Dordrecht.

Julie, C. (2006). Mathematical literacy: Myths, further inclusions, and exclusions. Pythagoras, 12(1), 62-69. doi: 10.4102/pythagoras.v0i64.100.

Kilpatrick, J. (2001). Understanding mathematical literacy: The contribution of research. Edu. Studies in Mathematics, 47, 101-116. doi: 10.1023/A:1017973827514.

Kramarski, B., \& Mizrachi, N. (2006). Online discussion and self-regulated learning: Effects of instructional methods on mathematical literacy. The Journal of Educational Research, 99(4), 218-231. doi: 10.3200/JOER.99.4.218-231.

Mkhwanazi, T. W., \& Bansilal, S. (2014). Mathematical literacy teachers' engagement with contextualized income tax calculations. Pythagoras, 35(2), 1-10. doi: 10.4102/pythagoras.v35i2.246.

Mumcu, H. Y. (2016). Using mathematics, mathematical applications, mathematical modelling, and mathematical literacy: A theoretical study. Journal of Education and Practice, 7(36), 80-96.

National Center for Education Statistics (1993). Adult literacy in America. Report of the National Adult Literacy Survey (NALS). Washington, DC: U.S. Department of Education.

National Center for Education Statistics. (2005). Highlights from the 2003 international adult literacy and life skills survey (ALL)-(Revised). Washington D.C.: National Center for Education Statistics (NCES).

Neubrand, M., Biehler, R., Blum, W., Cohors-Fresenborg, E., Flade, L., Knoche, N., \& Wynands, A. (2001). Framework zur Einordnung des PISA mathematik-Tests in Deutschland. Zentralblatt für Didaktik der Mathematik, 33(2), 45-60.

Nel, B. (2012). Transformation of teacher identity through a Mathematical Literacy re-skilling programme. South African Journal of Education, 32(2), 144-154.

Niss, M. A. (1999). Kompetencer og uddannelsesbeskrivelse. Uddannelse, 9, 21-29.

North, M., \& Christiansen, I. M. (2015). Problematizing current forms of legitimized participation in the examination papers for mathematical literacy. Pythagoras, 36(1), 285. doi: 10.4102/pythagoras.v36i1.285.

Noyes, A. (2017). Rethinking school mathematics. London: SAGE. 
OECD. (1999). Measuring student knowledge and skills: A new framework for assessment. OECD Publishing.

OECD. (2003). The PISA 2003 Assessment framework mathematics, reading, science, and problem-solving knowledge and skills. OECD Publishing.

OECD. (2006). PISA 2006 technical report. OECD Publishing.

OECD. (2009). PISA 2009 assessment framework key competencies in reading, mathematics, and science. OECD Publishing.

OECD. (2013). PISA 2012 assessment and analytical framework: Mathematics, reading, science, problem solving and financial literacy. OECD Publishing.

OECD. (2017). PISA 2015 assessment and analytical framework: science, reading, mathematics, financial, literacy and collaborative problem solving, revised edition, PISA. OECD Publishing.

Ojose, B. (2011). Mathematics literacy: Are we able to put the mathematics we learn into everyday use? Journal of Mathematics Education, 4(1), 89-100.

Reys, R. E., Suydam, M. N., Lindquist, M. M., \& Smith, N. L. (1998). Helping children learn mathematics. Boston: Allyn and Bacon.

Sáenz, C. (2009). The role of contextual, conceptual and procedural knowledge in activating mathematical competencies (PISA). Educational Studies in Mathematics, 71(2), 123-143. doi: 10.1007/s10649-008-9167-8.

Solomon, Y. (2009). Mathematical literacy: Developing identities of inclusion. New York: Routledge.

Spangenberg, E. D. (2012). Thinking styles of mathematics and mathematical literacy learners: Implications for subject choice. Pythagoras, 33(3), 1-12. doi: 10.4102/pythagoras.v33i3.179.

Stacey, K. (2011). The PISA view of mathematical literacy in Indonesia. Ind.MS, Journal on Mathematics Education, 2(2), 95-126. doi: 10.22342/jme.2.2.746.95-126.

Steen, L. A. (Ed.) (2001). Mathematics and democracy: The case for quantitative literacy. Princeton NJ: National Council on Education and the Disciplines.

Suharta, I., \& Suarjana, I. (2018). A case study on mathematical literacy of prospective elementary school teachers. International Journal of Instruction, 11(2), 413-424. doi: 10.12973/iji.2018.11228a.

Suryadi, D. (2012). Membangun Budaya Baru Dalam Berpikir Matematika. Rizqi Press. Bandung.

Umay, A. (2003). The ability of mathematical reasoning. Hacettepe University, The Journal of the Education Faculty, 24, 234-243.

Venkat, H. (2010). Exploring the nature and coherence of mathematical work in South African mathematical literacy classrooms. Research in Mathematics Education, 12(1), 53-68. doi: 10.1080/14794800903569865.

Venkat, H., Graven, M., Lampen, E., Nalube, P., \& Chitera, N. (2009). Reasoning and reflecting in mathematical literacy. Learning and Teaching Mathematics, 7, 47-53.

Yore, L. D., Pimm, D., \& Tuan, H. L. (2007). The literacy component of mathematical and scientific literacy. International Journal of Science and Mathematics Education, 5(4), 559-589. doi: 10.1007/s10763-007-9089-4. 